\title{
A New Feedforward Interference Cancellation using Bandstop Resonators for In-Band Interferers
}

\author{
Sarah Adel Ibrahim and Ronan Farrell
}

\begin{abstract}
High power wideband interferers appearing at 20, 15 and $10 \mathrm{MHz}$ away from the mobile RX band edge can now be suppressed by applying feedforward cancellation in the RF domain where the filtering is carried out using a new bandstop resonator. A study is conducted on the design of high selective resonators using power splitters, short-terminated delay lines and no attenuators. A 4-tap resonator is designed and measured showing a $3 \mathrm{~dB}$ bandwidth of $11 \mathrm{MHz}$ only, a maximum insertion loss of $3.9 \mathrm{~dB}$ and more than $21 \mathrm{~dB}$ rejection. The system is tuned to handle interferers at different locations using a voltage control phase shifter. The cancellation performance is tested using two $10 \mathrm{MHz}$ wide LTE modulated signals representing both the desired received signal $(-50 \mathrm{dBm})$ and the interferer $(-20 \mathrm{dBm})$. The power spectrum measurements show more than $45 \mathrm{~dB}$ cancellation for the interferers at 20 and $15 \mathrm{MHz}$, while $20 \mathrm{~dB}$ for the interferer at $10 \mathrm{MHz}$ frequency separation leaving the desired signal with less than $5 \mathrm{~dB}$ insertion loss and no significant noise is added.
\end{abstract}

Index Terms-Bandstop resonators, feedforward cancellation, in-Band interferers.

\section{INTRODUCTION}

O NE of the key challenges introduced with the new LTE spectrum fragmentation is the in-band interference, as a result of allocating many LTE bands in highly congested parts of the spectrum. A famous example is the Digital TV broadcasting and public safety signals whose transmitters operate at few $\mathrm{MHz}$ away from the LTE upper $\mathrm{C}$ band $(776 \mathrm{MHz}-787 \mathrm{MHz})$. These interference signals can reach the receiver with high power levels of up to $-20 \sim-15 \mathrm{dBm}$, which will not only degrade the quality of communication but also can cause severe impairments to the components of the receiver.

Gharpurey et al. [1] introduced a feed forward interferer cancellation technique which targets narrowband interferers only $(<2 \mathrm{MHz})$. In this technique, the desired signal is down converted to DC leaving the interferer at some intermediate frequency (IF). Another example showing a hybrid RF-DSP cancellation where the receiver linearity is much improved and wideband interferers can be controlled. However a successful cancellation is obtained for only large frequency offset interferers [2]. An exploration on the possibility of

Manuscript received May 9, 2016; accepted June 16, 2016. Date of publication August 26, 2016; date of current version October 5, 2016. This work was supported by the Science Foundation Ireland under Grant No.10/CE/I1853 as part of the Centre for Telecommunications Research (CTVR).

The authors are with the Department of Electronic Engineering, National University of Ireland Maynooth,

Color versions of one or more of the figures in this letter are available online at http://ieeexplore.ieee.org.

Digital Object Identifier 10.1109/LMWC.2016.2601292

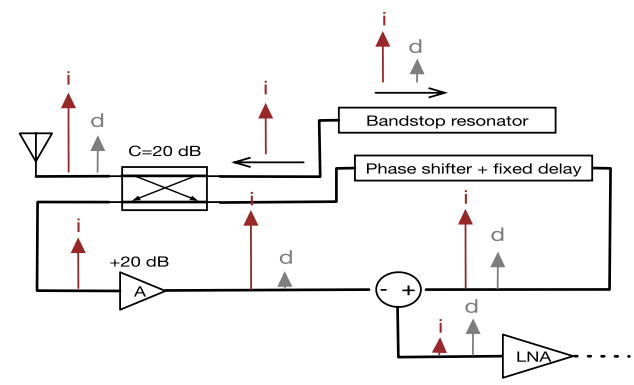

Fig. 1. The block diagram for the setup proposed to suppress out of band $\mathrm{RF}$ interference signals using bandstop RF resonators.

having a complete passive way of blocking interference at $\mathrm{RF}$ using N-path filtering is presented in [3]. This method is successful for only low power interferers as it still suffers from non-linearity (IIP3 $>17 \mathrm{dBm}$ ). In this letter a new feedforward technique is presented where both the filtering and the cancellation are implemented in the RF domain using a new high selective bandstop resonator. This work targets high power interferers with only few $\mathrm{MHz}$ away from the receiver.

\section{SySTEM OVERVIEW}

Fig. 1 shows the block diagram of the feedforward cancellation technique proposed in this letter using bandstop resonators. The received signal is first injected to a bi-directional $20 \mathrm{~dB}$ coupler whose coupling port is connected to a bandstop resonator, through port forms the main path and isolation port forms the auxiliary path. The interferer signal is reflected back from the resonator leaving the coupler at the isolation port where its amplified and subtracted from the main path. For this system to work, a very sharp resonator is required with high selectivity to separate the desired signal (d) from interferers (i) appearing at less than $20 \mathrm{MHz}$ away. In addition, the stop band must be wide enough to cover the total bandwidth of the desired signal to successfully eliminate the interferer while keeping the distortion levels for the received signal at minimum.

\section{Circuit Design And Simulations}

The design presented in this letter is based on the conventional structure of a 2-tap notch filter, which consists of a power divider and combiner joined by two different length transmission lines with a delay difference $(T)$ as introduced in [4]. In this letter, the power combiner is eliminated and a short circuit termination is added to each of the two taps. 


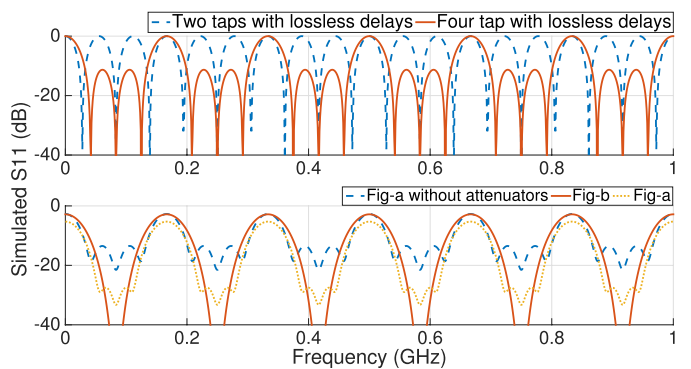

Fig. 2. The simulated S-parameter for the 2-tap and 4-tap resonator with a maximum delay of $9 \mathrm{~ns}$.

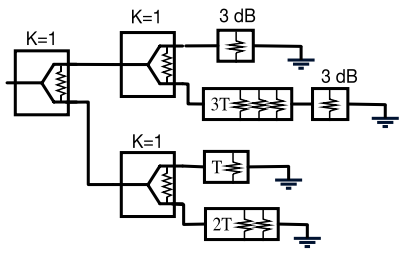

(a)

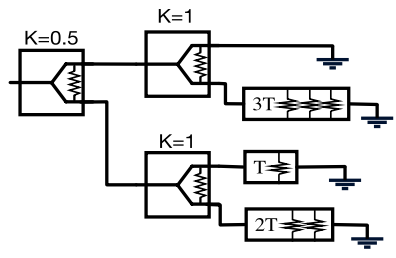

(b)
Fig. 3. The circuit model of the new 4-tap stop band resonator. a- with added attenuators, b- with unequal power split ratio.

The signals are now reflected at the short termination, recombined at the input and a single notch appears between every two peaks at frequencies $f_{\text {notch }}=\frac{n}{2 T}$, where $\mathrm{n}$ is an odd number. By increasing the number of taps (e.g. 4 taps) such that the time delay step at each tap is a multiple of $T$, multiple notches can be created in the frequency response between every two peaks (e.g. the four quarters of the signal are reflected out of phase). The first subplot in Fig. 2 shows the circuit simulation results of a 2-tap and 4-tap resonators constructed using ideal components from System-Passive library in Agilent ADS 2011. The delay elements of the first resonator was set to a value of $9 \mathrm{~ns}$, while the three delays of the 4-tap are set to $3 \mathrm{~ns}, 6 \mathrm{~ns}$ and $9 \mathrm{~ns}$. Having the same maximum delay for the two resonators, the peaks will appear at the same frequency. With this maximum delay value $\left(T_{\max }\right)$, an interferer at less than $27 \mathrm{MHz}\left(\frac{1}{4 T_{\max }}\right)$ can be attenuated.

Up till now, it is assumed that all signal taps carry the same power which will never be the case in practice. In fact, the power combiner will always receive unequal amplitude levels from the four delay taps. For the reason that, each delay will introduce a different insertion loss to the signal. But, as long as the insertion loss is directly proportional to the delay values, peaks and notches can still be formed. In this letter, a study is carried out on the effect of the amplitude of each tap on the frequency response. And, it is noticed that by lowering the amplitude of the signals reflected back from the taps with the smallest and largest delays, the attenuation response can be improved. This can be demonstrated by inserting additional attenuators (e.g. $3 \mathrm{~dB}$ ) to the first and fourth signal tap as shown in Fig. 3-a. The circuit simulations are again repeated for the 4-tap model with/without the attenuators in the second subplot of Fig. 2, where more than $14 \mathrm{~dB}$ extra attenuation appears for the second case, leaving the pass band with an additional losses of $2.5 \mathrm{~dB}$.

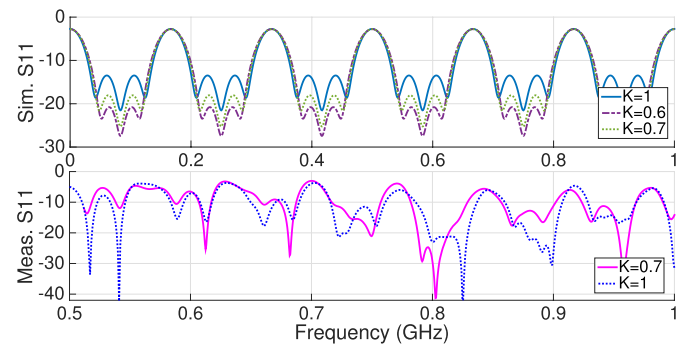

Fig. 4. The simulated S-parameter for 4-tap bandstop resonators with different power split ratios, $T=3$ ns.

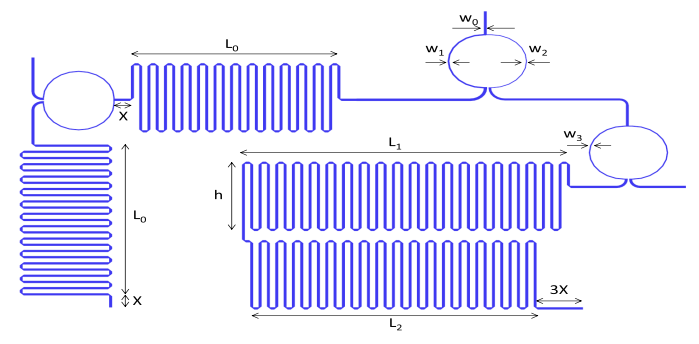

Fig. 5. The board layout of the 4-tap band stop resonator with $\mathrm{K}=0.7$. The outline of the board is $13.5 \mathrm{~cm} \times 22.5 \mathrm{~cm}$.

In order to avoid these extra losses, it is suggested to adjust the power split ratio of the first power divider at the input port instead as shown in Fig. 3-b. The power split ratio $(\mathrm{K})$ is the ratio between the power at the first output to that in the second output. Therefore, $\mathrm{K}=0.5$ suggests distributing one third of the signal among the two taps with the largest and smallest delays leaving the rest of power equally distributed among the two other taps. Fig. 4 shows the circuit simulation results of the modified resonator with different values for the unequal power split ratio $(\mathrm{K})$.

\section{Microstrip Implementation And MeAsurements}

Here, the 4-tap bandstop resonator circuit is implemented using Microstrip technology. Ceramic substrate (Rogers 6010) is selected with high prematurity $\left(\varepsilon_{r}=10.2\right.$, thickness $=1.27 \mathrm{~mm}$ ) to obtain the large delays required. Meander transmission lines with $50 \mathrm{ohm}$ impedance are constructed such that the height is less than quarter the propagation wavelength $(28 \mathrm{~mm})$ to suppress higher order modes. The lines are separated by not less than $2 \mathrm{~mm}$ to reduce coupling losses. The maximum required delay is calculated for a frequency offset of $20 \mathrm{MHz}$ with the interferer signal centered at $770 \mathrm{MHz}$ to be $\tau=7.3 \mathrm{~ns}, 14.6 \mathrm{~ns}$ and $21.9 \mathrm{~ns}$ delay for the three taps. The four lines are then terminated with an open circuit rather than a short to facilitate the fabrication process and avoid the losses added by grounded vias.

Unequal power split ratio for the first power divider is used with $\mathrm{K}=0.7$, which is the smallest possible value for having the line dimensions of greater than $0.2 \mathrm{~mm}$ for the output ports. Fig. 5 shows the board layout for the resonator with $\mathrm{K}=0.7$, and Table. I lists all the different dimensions for the lines. Fig. 4 shows a comparison between the measured $\mathrm{S}$-parameters of the fabricated circuits. Measurements shows 
TABLE I

GeOMETRic Dimensions of THE BoARd In mm

\begin{tabular}{|c|c|c|c|c|c|c|c|c|c|}
\hline$w_{0}$ & 1.367 & $w_{2}$ & 0.281 & $L_{0}$ & 69.3 & $L_{2}$ & 97.8 & $h$ & 30.5 \\
\hline$w_{1}$ & 0.772 & $w_{3}$ & 0.497 & $L_{1}$ & 108.5 & $s$ & 1.75 & $X$ & 5.0 \\
\hline
\end{tabular}

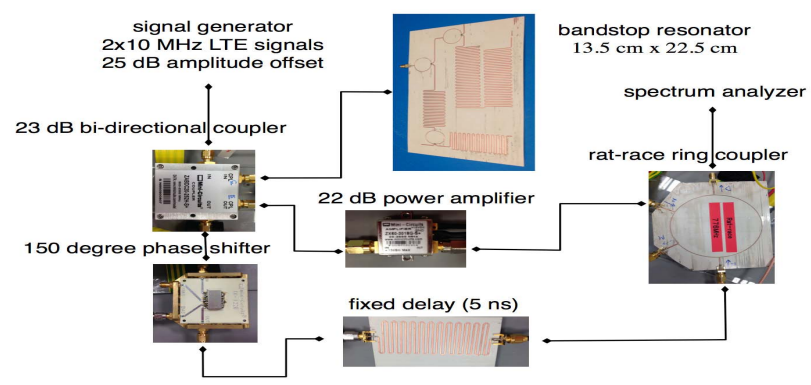

Fig. 6. The test bench for the new interference cancellation with a 4-tap stopband resonator $(\mathrm{K}=0.7)$.

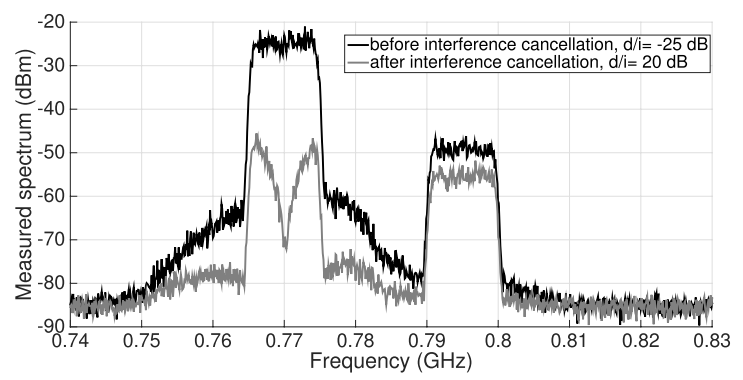

Fig. 7. The measured power spectrum for the received signals before and after interference cancellation with (i) at $770 \mathrm{MHz}$ and (d) at $795 \mathrm{MHz}$.

a good agreement with the circuit simulation at frequencies around $770 \mathrm{MHz}$ which is the frequency on which the Wilkinson power splitters are designed. To obtain this agreement on a wider band of frequency, the power splitters must be designed with multiple sections of quarter wave transformers. The measured resonator with unequal power split ratio shows $2 \mathrm{~dB}$ less insertion loss compared to the resonator designed with equal power ratio.

\section{Test Results}

Fig. 6 shows the setup of the test bench used to test the interference cancellation using the new fabricated bandstop resonator with $\mathrm{K}=0.7$. A fixed microstrip delay of $5 \mathrm{~ns}$ together with a voltage controlled phase shifter are used to compensate for the phase/delay in the feedforward path. Two LTE modulated signals are generated with a bandwidth of $10 \mathrm{MHz}$ for each. The interferer $(-25 \mathrm{dBm})$ is first located at $770 \mathrm{MHz}, 20 \mathrm{MHz}$ away from the edge of the receiver $(-50 \mathrm{dBm})$. The power spectrum measurements are first recorded for the signal at the input of the bi-directional coupler, then at the output of the rat race ring coupler. More than $45 \mathrm{~dB}$ cancellation is measured at the center of the interferer, beside more than $25 \mathrm{~dB}$ cancellation over

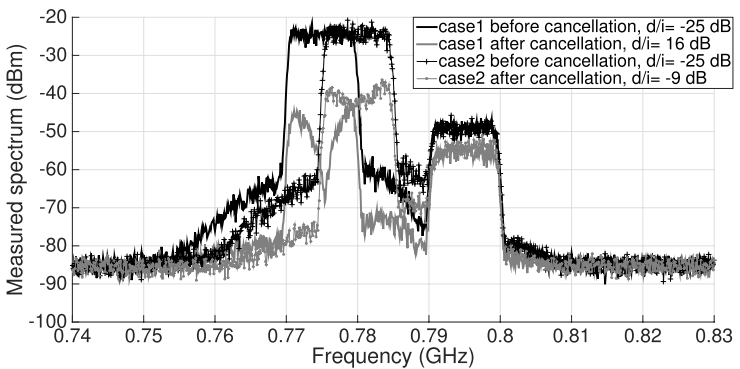

Fig. 8. The measured power spectrum for the received signals before and after interference cancellation with (d) at $795 \mathrm{MHz}$, (i) at 15 and $10 \mathrm{MHz}$ offset.

TABLE II

Summary of the Measured Parameters and COMParison

\begin{tabular}{|c|c|c|c|c|}
\hline & ref. [1] & ref.[2] & ref. [3] & This work \\
\hline \hline Frequency offset(MHz) & 23 & 21 & $>60$ & $15-10$ \\
\hline Rejection(dB), BW(MHz) & $20,<1$ & 24,8 & 18,6 & 23,10 \\
\hline P-1dB, IIP3 (dBm) & $-29.5,-$ &,-- & $6,>17$ & $12.8,>25$ \\
\hline
\end{tabular}

the $10 \mathrm{MHz}$ band as seen in Fig. 7, which also points to the desired signal to interference power ratio before and after cancellation (d/i). Measurements are again repeated with the frequency separation reduced to 15 and $10 \mathrm{MHz}$, where in each case the voltage controlled phase shifter is slightly tuned. A successful cancellation for the interferer is again obtained leaving the receiver band slightly attenuated by less than $5 \mathrm{~dB}$ as shown in Fig. 8. Furthermore, a comparison is conducted in Table II showing a high rejection for wideband interferers at smaller frequency offsets, in addition to a higher $1 \mathrm{~dB}$ compression point when compared to other solutions.

\section{CONCLUSION}

In this letter, a study is presented on the design of new bandstop resonators with high selectivity for in-band interference cancellation. A 4-tap resonator is fabricated and measured showing a $3 \mathrm{~dB}$ bandwidth of $11 \mathrm{MHz}$ only, a maximum insertion loss of $3.9 \mathrm{~dB}$, in addition to $>21 \mathrm{~dB}$ rejection. A test is conducted using two $10 \mathrm{MHz}$ LTE signals: (i) with an average peak power of $-25 \mathrm{dBm}$; and (d) with $-50 \mathrm{dBm}$. Three cases with different frequency offsets are tested: $20 ; 15$; and $10 \mathrm{MHz}$. More than $45 \mathrm{~dB}$ cancellation is achieved for the first two cases, and $20 \mathrm{~dB}$ for the third case.

\section{REFERENCES}

[1] S. Ayazian and R. Gharpurey, "Feedforward interference cancellation in radio receiver front-ends," IEEE Trans. Circuits Syst. II, Exp. Briefs, vol. 54, no. 10, pp. 902-906, Oct. 2007.

[2] M. W. Wyville, R. C. Smiley, and J. S. Wight, "Frequency agile RF filter for interference attenuation," in Proc. IEEE Radio Wireless Symp., Jan. 2012, pp. 399-402.

[3] A. Ghaffari, E. Klumperink, and B. Nauta, "8-path tunable RF notch filters for blocker suppression," in Proc. IEEE Int. Solid-State Circuits Conf., Feb. 2012, pp. 76-78.

[4] K. Gerald I, "Tunable microwave notch filter," U.S. Patent 3895304 A, Jul. 15, 1975. 